\title{
Les ambivalences de l'animation dans un parc à thème pour enfants, entre jeu et éducation
}

The ambiguities of animation in a theme park for children, between play and education

\section{Sophie Corbillé}

\section{OpenEdition}

Journals

Édition électronique

URL : https://journals.openedition.org/sdj/2274

DOI : $10.4000 /$ sdj.2274

ISSN : 2269-2657

Éditeur

Laboratoire EXPERICE - Centre de Recherche Interuniversitaire Expérience Ressources Culturelles Education

\section{Référence électronique}

Sophie Corbillé, « Les ambivalences de l'animation dans un parc à thème pour enfants, entre jeu et éducation », Sciences du jeu [En ligne], 12 | 2019, mis en ligne le 19 décembre 2019, consulté le 12 décembre 2022. URL : http://journals.openedition.org/sdj/2274; DOI : https://doi.org/10.4000/sdj. 2274

Ce document a été généré automatiquement le 12 décembre 2022.

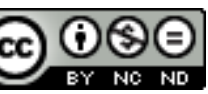

Creative Commons - Attribution - Pas d'Utilisation Commerciale - Pas de Modification 4.0 International - CC BY-NC-ND 4.0

https://creativecommons.org/licenses/by-nc-nd/4.0/ 


\section{Les ambivalences de l'animation dans un parc à thème pour enfants, entre jeu et éducation}

The ambiguities of animation in a theme park for children, between play and education

Sophie Corbillé

1 La place des enfants dans les grandes villes a évolué ces dernières décennies. D’un côté, l'usage des espaces publics, en particulier celui de la rue où les enfants ont longtemps joué, s'est réduit, notamment pour des raisons de sécurité auxquelles les parents sont particulièrement attentifs, mais aussi plus largement du fait des politiques urbaines d'inspiration néo-libérale qui encouragent la privatisation des espaces publics (Chombart de Lawe et al., 1976 ; Katz, 2004 ; Wridt, 2004 ; Else, 2009 ; Authier et LehmanFrisch, 2012; Sullu, 2018). De l'autre, de nouveaux lieux plus organisés et institutionnalisés se sont multipliés, proposant des activités de toutes sortes: parfois très encadrées et d'autres fois peu, elles sont souvent à la croisée du ludique et de l'éducatif. Parmi ces lieux, on trouve des espaces et des structures publiques ou sans but lucratif: bibliothèques, ludothèques, accueils collectifs de mineurs, musées des enfants, etc. Leur place est plus ou moins importante selon les pays et la façon dont les États et les organismes publics considèrent cette mission. En France, les mouvements d'éducation populaire ont joué un rôle crucial dans leur développement (Chombart de Lawe et al., 1976; Roucous, 2006). Mais on trouve également, et ils sont de plus en plus nombreux, des espaces créés par le secteur privé, caractérisés cette fois par une dimension commerciale et des enjeux de rentabilité évidents, posant la question de la «marchandisation » de l'enfance (McKendrick et al., 2000; Horschelman et van Blerk, 2011 ; Sullu, 2018) : parcs d'attractions en plein air ou fermés, espaces de jeux d'évasion [escape game] ou de jeu laser [laser game], ou encore mini-villes, voilà quelques exemples de lieux payants qui proposent d'occuper et de divertir les enfants pendant leur temps libre. C'est à l'une de ces mini-villes, appelée KidZania, que cet article est consacré1. 


\section{Un parc nouvelle génération : entre jeu, commerce et éducation}

2 KidZania est une entreprise mexicaine, qui a ouvert son premier parc en 1999 à Santa $\mathrm{Fe}$, dans la ville de Mexico. Depuis, le groupe s'est internationalisé: il possède aujourd'hui une trentaine de parcs franchisés ${ }^{2}$ sur tous les continents, toujours situés dans des centres commerciaux construits dans les quartiers chics de grandes villes, et dans lesquels plus de soixante millions de visiteurs se seraient déjà rendus ${ }^{3}$. Dans ces parcs, les enfants sont invités à jouer à travailler en choisissant des métiers parmi plusieurs dizaines de jeux de rôle : pilote, dentiste, caissier, laveur de voiture, livreur de colis, journaliste, présentateur télé, etc. Les parcs obéissent tous à un même modèle. Construits comme une ville miniature, les rez-de-chaussée des édifices qui bordent les rues sont occupés par une "vraie » entreprise locale, régionale ou globale, et aussi, mais en nombre moins important et variant selon les pays, par quelques institutions publiques. Ce sont dans ces "établissements» ou «locaux» que les jeux de rôles, appelés "activités ", se déroulent en grande partie. À l'entrée du parc, une somme d'argent en "KidZo", la monnaie du lieu, est remise aux enfants sous forme d'un chèque. Après un passage à la banque pour recevoir des espèces, ils peuvent accroître leur pécule en travaillant dans les espaces "gérés" par les entreprises et les institutions. Un ou plusieurs animateurs, appelés Zupervisors, les reçoivent et leur présentent le métier qu'ils vont exercer et pour lequel ils seront rémunérés. L'argent peut être dépensé s'ils achètent un bien ou un service dans ces mêmes établissements : se faire poser un plâtre à la clinique, se payer une formation d'apprentis pilote, se rendre en discothèque, passer le permis de conduire ou obtenir un diplôme à l'université.

3 Les parcs affichent une vocation éducative, se positionnant sur le secteur du divertissement éducatif (edutainement) qui connait une forte croissance depuis plusieurs années : «En mélangeant la réalité avec l'amusement, KidZania propose une expérience de développement authentique et puissante, qui prépare les enfants à comprendre et à organiser leur monde ${ }^{4}$ " peut-on lire sur le site. L'entreprise indique par ailleurs transmettre des valeurs, comme «la créativité, la pensée critique, la communication, la confiance et la collaboration ${ }^{5}$ ». À cela s'ajoute enfin une dimension citoyenne, liée à «l'histoire » de KidZania telle qu'elle est contée par le groupe et dans ses parcs, à la manière d'un mythe fondateur.

Dans un monde traversé par les inégalités, des enfants auraient créé KidZania, nation régie par les droits «à être, à partager, à créer, à savoir, à prendre soin, à jouer ${ }^{6}$ ». Aux figures du travailleur et du consommateur, s'ajoute ainsi celle du citoyen œuvrant à l'élaboration d'un monde meilleur, incarné par le slogan du lieu: «Sois prêt pour un meilleur monde ${ }^{7}$ ». On a donc là un espace de divertissement privé, à vocation lucrative, très imbriqué avec le monde économique en raison de la présence de marques et d'entreprises, et qui propose de participer à l'éducation des enfants en les familiarisant avec le travail, l'argent, mais également, bien que de manière plus marginale, avec des enjeux citoyens. C'est notamment le cas du parc de Cuicuilco au Mexique où des activités sur le handicap, l'écologie et la citoyenneté ont été développées. Par ailleurs, l'Instituto Nacional Electoral, Le Ministerio Público et le Servicio de Administración Tributaria (service des impôts mexicain) y sont présents, chargés de transmettre des comportements citoyens, comme : payer des taxes et des impôts, respecter la loi ou 
encore voter. Des activités de type culturel sont aussi parfois proposées en complément dans les parcs de l'entreprise. À titre d'exemple, à Dubaï en 2017, un Heritage village avait été temporairement installé : les enfants, dans un décor évoquant le mode de vie bédouin, pouvaient se familiariser avec quelques traditions locales comme la poterie ou la pratique du henné ${ }^{8}$.

5 La réunion du jeu et de l'apprentissage, pour autant que ces notions puissent sembler antinomiques et que le terme anglais edutainement leur confère un caractère de nouveauté, ne date cependant pas d'aujourd'hui. Gilles Brougère rappelle ainsi que «l'association moderne qui voit dans le jeu un potentiel développemental et éducatif s'enracine [en réalité] dans la pensée romantique. » (Brougère, 2002, p. 6). De même, Baptiste Besse-Patin (2019), en s'appuyant sur des travaux menés dans des pays occidentaux, souligne que la contamination du loisir par l'éducation est ancienne, et qu'elle s'accompagne de la création d'institutions qui, peu à peu, ont pris en charge les activités extra-scolaires, participant d'une véritable "rentabilité éducative » qui se niche jusque dans les loisirs des enfants. Bien évidemment, les espaces ludiques contemporains offrent des situations variées dans lesquelles le jeu, tout comme l'éducation, prennent des formes différentes, allant d'activités très libres et parfois sans but précis si ce n'est celui de jouer, à d'autres plus encadrées et tendues vers un objectif de développement, souvent supervisées par des adultes. Qu'en est-il dans les parcs KidZania qui proposent aux enfants de découvrir le travail à travers des jeux de rôles, dans un espace construit autour et avec de "vrais » acteurs économiques et publics ? À partir des pratiques et des modalités d'intervention des animateurs, nous chercherons à analyser comment ces professionnels procèdent pour faire entrer les enfants dans ces jeux à vocation éducative. Mais nous nous poserons aussi la question du côté des enfants pour donner à voir comment ils se saisissent de ces propositions. Bien sûr, une étude du dispositif est nécessaire en amont afin de comprendre quel jeu leur est proposé. Autrement dit, il s'agira de réfléchir à la façon dont les animateurs sont amenés à faire un travail de médiation entre les dispositifs du parc qui constituent le cadre de leur activité, et la réception que peuvent en avoir les enfants qui, il faut le souligner, ne constituent pas un public homogène, en raison leurs différences d'âge (entre 4 ans et 14 ans environ ${ }^{9}$ ), et plus banalement de la singularité de chacun d'entre eux.

6 Cet article repose principalement sur une enquête de terrain conduite dans le parc de Santiago du Chili où une dizaine de demi-journées d'observation ont été menées, à suivre des groupes d'enfants dans le cadre de sorties familiales ou avec d'autres familles, entre 2016 et $2018^{10}$. L'attention a été portée sur le parc dans son ensemble: l'organisation matérielle et symbolique du lieu, la foule des enfants, les interactions entre eux, avec leurs parents et avec les animateurs, les échanges de paroles, et les objets qui circulent et qui sont fabriqués. De manière classique en ethnographie, il s'est agi d'observer et de décrire : prise de notes sur le téléphone, photographies, collecte de documents dans le parc et tenue d'un journal de terrain.

7 Ce travail d'observation et de description permet de mettre au jour des régularités dans les logiques d'action et notamment d'animation. Une limite est à souligner cependant : les parents et adultes ne sont pas admis dans les établissements. Ils peuvent certes observer les enfants à travers les vitres, mais n'entendent pas les échanges entre l'animateur et ces derniers. Une série d'entretiens ont également été conduits. Deux avec des animatrices qui ont travaillé dans le parc: l'une avait la charge des 
anniversaires ${ }^{11}$, et l'autre, Camila, était responsable d'activités et de jeux de rôles dans différents établissements. Ce dernier entretien, très approfondi, explicite particulièrement bien les observations concernant les pratiques des animateurs dans le parc et occupe à ce titre une place importante dans l'analyse développée ici. Un entretien a également été mené avec un des gérants du parc, et cinq autres avec des parents que j'ai accompagnés ou qui avaient fréquenté le parc précédemment. Ce terrain a été complété par un autre conduit dans le parc de Dubaï : cinq demi-journées d'observation se sont déroulées avec des familles d'expatriés résidant à Abou Dhabi et Dubaï entre 2016 et 2019, suivies d'entretiens avec les parents. Pour finir, l'analyse prend en compte les discours produits par l'entreprise sur ses sites internet et les réseaux sociaux ${ }^{12}$. Les enfants que j'ai accompagnés, et qui sont au centre de ces deux terrains, ont entre cinq et dix ans.

L'analyse reposera sur une approche pragmatique des activités d'animation, attentive aux manières de faire, aux interactions et à leur cadre, dans la continuité de travaux menés sur l'animation (Roucous, 2007 ; Besse-Patin, 2019). Il s'agira de privilégier « une perspective culturelle » des pratiques d'animation et de jeu (Roucous et Haberbusch, 2010), sans chercher à évaluer les processus et les facteurs, formels ou informels, d'apprentissage dans le jeu. En outre, les activités se déroulant dans un parc d'attractions, la réflexion s'appuiera sur des recherches produites sur ces espaces, attentives au dispositif matériel et narratif, aux interactions qui s'y déploient, ou encore à l'organisation du travail qui les régit. Comme le rappelle Thibaut clément (2016) qui a consacré un ouvrage aux parcs Disney, les parcs à thèmes ont donné lieu à de très nombreuses études depuis les années 1970, menées par des chercheurs issus de disciplines diverses. Deux grandes approches se dessinent à leur lecture. La première (1970 - fin des années 90) s'inscrit dans une perspective sémiotique : elle considère les parcs comme " un "discours", un ensemble de représentations dépourvues de signifiés et entretenant volontairement la confusion entre le réel et l'imaginaire, [faisant] du parc le symbole par excellence de la condition postmoderne de l'homme contemporain. » (Clément, 2016, p. 11) Autrement dit, il s'agit, dans une perspective critique, de percer à jour la manière dont l'idéologie est mise en espace et en récit. Parmi ces travaux, on peut citer ceux de Marin (1973), Baudrillard (1981), Eco (1987), ou encore Fjellman (1992). La seconde approche, plus récente, et qui s'est développée en réaction aux recherches précédentes et à la faveur de la montée en puissance de la microsociologie et de l'interactionnisme, "accorde au visiteur un plus large degré de liberté, assimilant le parc à un espace de jeu ou de performance théâtrale dont le script et les règles implicites seraient en permanence renégociés » (Clément, 2016, p. 14). Ici, le regard est porté sur les pratiques des visiteurs, sur les interactions avec l'environnement (Moore et al., 1980; Augé 1997) et les professionnels qui travaillent dans les lieux et animent les jeux (Van Maanen, 1991 ; Chesneaux 1997). Depuis le début des années 2000, des travaux cherchent néanmoins à dépasser ces deux approches en s'intéressant non seulement à la façon dont ces lieux sont construits et à ce qu'ils font aux visiteurs, mais aussi à ce que les visiteurs en font. C'est par exemple le sens de la recherche de Thibaut clément qui s'intéresse à la fois aux " procédés de fabrication des parcs et [aux] discours entourant leur conception" et aux "usages effectifs des visiteurs et des employés dans l'espace de parcs ». (Clément, 2016, p. 17-18). Mon enquête s'inscrit également dans cette double attention.

Entendue comme une activité sociale et symbolique, l'animation se situe dans un espace-temps donné. Dans l'exploration de cette activité, il importe donc de se pencher 
d'abord sur son cadre matériel et symbolique, qui constitue le cadre de l'animation : la façon dont l'espace est aménagé, de même que les objets qui sont à disposition des animateurs et des enfants. Nous nous intéresserons ensuite aux façons de faire des animateurs, et aux relations qu'ils ont avec les enfants, les menant à opérer des $(\mathrm{re})$ cadrages permanents pour animer les jeux de rôle et (s')» enrôler ». Nous verrons en quoi cela contribue à définir une manière de jouer particulière, qui pose la question des tensions entre les intentions éducatives et la dimension de divertissement, dans un contexte où les intérêts privés sont très présents. Enfin, nous montrerons comment le contexte de travail d'une industrie du divertissement, éducatif s'il en est, participe à façonner en parallèle les pratiques d'animation.

\section{L'aménagement d'une ville à jouer}

10 Le site de Santiago a été inauguré en 2012 à Las Condes, une commune cossue de la capitale chilienne où de nombreuses activités économiques se sont installées, donnant naissance à "Sanhattan", quartier d'affaires et financier à cheval sur plusieurs communes. Il est situé dans le parc public Araucano, l'un des plus grands espaces verts de la ville, à proximité d'un vaste centre commercial, d'édifices résidentiels et d'affaire et d'un club de golf. Comme le directeur du parc rencontré l'indique, cette localisation permet d'être au plus près des flux de personnes. Environ 380000 visiteurs se rendent chaque année au parc, $55 \%$ d'enfants et $45 \%$ de parents. Ils appartiennent majoritairement aux segments aisés de la population ${ }^{13}$.

11 Le parc a pour décor une ville miniature, construite à partir d'éléments urbains reconnaissables, appartenant cependant à un imaginaire largement européen et parfois très éloigné des villes dans lesquelles les enfants qui fréquentent le parc résident: pavés, lampadaires, ou encore place avec son théatre et son hôtel de ville surmonté d'une horloge. L'espace est saturé par toutes sortes de signes (signalétique urbaine et noms des marques et des entreprises), par les lumières des enseignes et par le niveau sonore élevé de la musique diffusée qui se mêle aux paroles et cris des enfants. Emilia, qui réside dans une commune proche de Santiago (8 ans et demi) décrit ainsi le lieu: " Il y a une clinique, un bar, une université, tout ce que les adultes font. Il y a Banco de Chile, Chilexpress, une pharmacie, une pizzeria. Ça ressemble à une petite ville, comme à Santiago, mais pour les enfants. » Les parents suivent de près ou de loin leurs enfants selon l'âge de ceux-ci et l'envie de ces derniers de leur échapper. Ils sont en couple, seul ou avec des amis, souvent munis de leur téléphone dont ils font un usage intense. D'autres, moins nombreux, s'installent dans un café qui leur est réservé. Ils pourront ensuite localiser leurs enfants grâce au bracelet mis au poignet de tous à l'entrée.

Une ambiance bruyante et agitée caractérise le parc. Outre la fréquentation importante lors des observations et l'entrain des enfants, cela tient en partie à la densité de la soixantaine d'activités. La majorité est gérée par les partenaires de KidZania, des groupes privés régionaux, nationaux ou mondiaux. On ne peut manquer leur présence en raison des enseignes qui portent leur nom: Banco de Chile, Prosegur, Entel, Nivea, Clinica Alemana, Radio Disney, Jumbo ou encore Manpower. Sont aussi présentes à

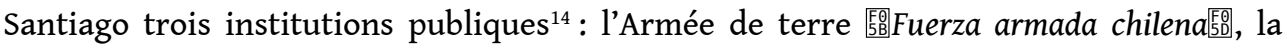

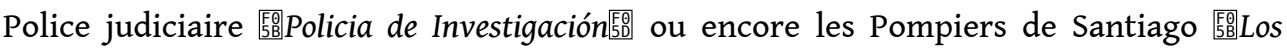
bomberos de Santiago 
13 La construction d'un parc franchisé se fait toujours en étroite collaboration avec la maison mère, et certaines grandes lignes sont à respecter. Outre l'aspect global de la ville, il y a l'obligation de retrouver partout certaines activités ou métiers - comme celui de pompier, de vétérinaire, de caissier ou encore de dentiste -, ou certaines entreprises, en particulier une compagnie aérienne du pays d'implantation. Celle-ci occupe une place importante dans la mise en récit du lieu : l'entrée du parc évoque un hall d'embarquement et la sortie, surplombée par un vrai nez d'avion, se présente comme le «bureau de l'immigration ». Cet imaginaire du voyage et de la frontière est au service de la fabrication de ce territoire de l'enfance qui se présente, on l'a vu, comme une nation indépendante. Il justifie par ailleurs la vente (en «vrai » argent cette fois) du passeport de KidZania ${ }^{15}$. Quoi de plus international, dans sa forme, qu'un aéroport, et de plus répandu que les métiers de pompier, dentiste ou caissier dans les grandes villes? Souvent globalisés, ces incontournables participent certainement à façonner des imaginaires enfantins globaux, puisque déclinés dans tous les parcs. Mais on trouve aussi des institutions et des entreprises locales que seuls les résidents du pays sont en mesure de connaître.

14 L'assortiment de ces acteurs privés et publics est au cœur du concept : être le plus « réel» possible. Selon les concepteurs et gérants, leur présence permet en effet de donner à voir une « vraie » ville avec de « vrais » espaces de travail, de consommation et de divertissement ${ }^{16}$. À cet égard, il faut souligner les efforts déployés pour donner un cadre aux jeux de rôle. Outre les déguisements systématiques pour chaque activité, les lieux sont aménagés de sorte à évoquer les espaces de travail, à travers un mobilier et des objets aisément reconnaissables: mini-chaîne de production dans la fabrique de sodas, de yaourts et de chocolats, ordinateurs et presse dans les locaux du journal, caméras, prompteurs et fond vert dans le studio télévisé, casques, écrans et micros à la radio, fauteuil surmonté de sa radio numérique et instruments divers chez le dentiste, caddies, boites alimentaires et caisses au supermarché, perruques et maillet à la cour de justice ou encore colis en carton et diable à la poste. Sans oublier l'argent, qui circule sous forme de billets, et qui peut être placé sur un compte, donnant droit alors à une carte bancaire. "À KidZania, tu dois travailler et ils te paient », explique Emilia, tandis que son frère range sa carte dans son portefeuille, en attendant peut-être d'en faire usage plus tard au distributeur automatique du parc. Lors des échanges avec les enfants, ceux-ci disent souvent avoir été saisis par cet effet de réel, notamment lors de leur première visite : ils se rappellent le bidon de gaz à transporter, la presse d'où sort le journal qu'ils ont fabriqué, le plâtre qu'on leur a posé, ou encore la pâte sur laquelle ils ont composé leur pizza.

15 En fait, la ville n'est bien sûr pas réelle. Le parc offre au contraire une ville idéalisée car débarrassée de tous ses "problèmes": sans ségrégations, sans pollution, sans pauvreté. Les études menées sur les parcs à thème montrent que le motif de la réalité est récurrent dans ce type de lieux. Chez Disney, par exemple, les Imachineurs, ces ingénieurs chargés de la conception des attractions, parlent de "réalité accrue »: il s'agit pour eux, comme le rappelle Clément, de

dépeindre le monde non tel qu'il est, mais tel qu'il devrait être, purifié des accidents et des contingences de l'histoire et donc révélé sous sa forme abstraite et originale. La « réalité accrue » s'avère alors un projet normatif qui, en autorisant les Imachineurs à discriminer entre les traits essentiels et accidentels de la réalité et en débarrassant cette dernière de ses errements, s'emploie à présenter le monde comme une marche linéaire et sans heurts vers le progrès (Clément, 2016, p. 54). 
Dans le cas de Kidzania, la réalité accrue conduit au paradoxe de faire (jouer à) travailler les enfants alors que c'est précisément interdit dans la vraie vie (Corbille, 2018). Comme de nombreux chercheurs travaillant sur le jeu l'ont montré, ce décalage vis-à-vis du «réel» est en fait une condition pour que le jeu se déploie. Gregory Bateson (1988 [1979], p. 132-133) note ainsi :

Ce qui est propre au jeu, c'est que ce terme désigne des contextes dont les actes constituants ont une pratique et une réalisation différentes de celles qu'elles auraient dans le non-jeu [...]. Il se pourrait même que l'essence du jeu réside dans la dénégation partielle de la signification qu'auraient dans d'autres situations les actes du jeu.

Les enfants ne sont d'ailleurs pas dupes et savent qu'ici «c'est pour de faux ». « Notre patient est mort trois fois ", raconte en riant un enfant d'une dizaine d'années qui sort de la clinique. Bref, le parc est davantage du côté de la vraisemblance que du vrai et ouvre un cadre de "second degré » (Brougère, 2005), essentiel aux pratiques de jeu et de loisir. Il s'agit moins de reproduire le réel que de le représenter et de le symboliser.

En raison de sa forme et des imaginaires convoqués, cet espace à animer et à jouer, extrêmement pensé, propose ainsi un "environnement suggestif» (Roucous et Haberbusch, 2010, p. 205) favorable à une entrée dans le jeu pour les enfants, et qui constitue une sorte «d'appel à jouer » (p. 200). À cela s'ajoutent les objets qui sont dans le parc, avec lesquels les enfants interagissent: voiture, pneus et outils du garagiste, lance à eau du pompier, calepin du journaliste, micro et casque de l'animateur radio, caddy du client, tableau de bord du pilote ou encore pompe à essence du pompiste. Comme l'explique Brougère $(2003,2013)$, il existe une rhétorique des objets qui intègrent des signes, des valeurs et des actions dans leur matérialité. Bien évidemment, celle-ci n'est pas donnée une fois pour toutes, et elle dépend du contexte et des individus qui s'en saisissent. Reste que « le jouet, par sa forme même et les images qu'il porte, met à disposition (afford en anglais) de l'enfant des actions possibles que la simple perception permettra d'activer. L'enfant décode les affordances de l'objet qui le conduisent à agir » $(2003$, p. 80$)$. Le parc est de ce point de vue avant tout une ville à jouer.

19 Cette approche n'est pas sans faire penser à la théorie de l'acteur-réseau qui s'attache à comprendre l'impact de la technique sur les pratiques et montre que les objets manufacturés, « dépositaires des savoirs et des représentations du monde [...] agissent en retour sur leurs usagers : de même qu'ils requièrent pour leur bon fonctionnement certaines manœuvres, de même les objets "font-ils faire" (Latour 2007, p. 316) des actions aux personnes qui les emploient. " (Clément, 2016, p. 20-21). A KidZania, les objets miniaturisés apparaissent comme des éléments clé des jeux de rôles, accompagnant, voire initiant le jeu, et le fixant inversement dans certains cas « dans des scénarii limités et restreints par les interactions programmées" (Roucous et Haberbusch, 2010, p. 204). Néanmoins, ici et là, quelques détournements s'observent, à l'instar du diable de l'entreprise DHL qui permet de livrer les colis, et dont les enfants, dans les allées de la ville et entre deux boutiques, se servent parfois comme d'un engin leur permettant de faire la course avec un compagnon de jeu.

20 L'espace du parc se présente ainsi comme un contexte aménagé dans lequel les animateurs vont animer des « jeux de rôles » et des « activités». Comment le font-ils ? Comment travaillent-ils ? Et quel(s) jeu(x) se déploie(nt)? 


\section{Jeu d'enrôlement et pas de côté}

21 Camila avait 19 ans quand elle a travaillé comme Zupervisors au KidZania de Santiago, peu après l'ouverture du parc, pendant cinq mois, environ quatre heures par jour, du lundi au vendredi. Comme beaucoup d'autres animateurs, elle était alors étudiante. Elle connaissait le lieu pour l'avoir fréquenté plusieurs fois avec sa petite sœur. Rétrospectivement, elle explique son envie d'y travailler par son goût pour l'enfance et le plaisir qu'elle a à être avec des petits :

On m'a toujours comparée à Peter Pan, car la petite fille en moi est toujours bien présente. 監.... Quand ils ont des problèmes, ce sont des problèmes simples, c'est facile de les aider à les résoudre. Ils adorent jouer, et même les enfants de 17 ans! Je me souviens qu'ils étaient heureux dans le parc, parce que même s'il s'agit de travailler, KidZania est en fait un immense terrain de jeu.

Après une formation d'une vingtaine d'heures, elle a été affectée à une "aire " supervisée par un "coordinateur", réunissant plusieurs "marques». Elle était alors responsable d'une activité, seule ou avec un collègue : accueil des enfants, animation du jeu de rôle qui repose sur un script précis écrit en accord avec l'entreprise ou l'institution concernée, mais aussi propreté du local, et comptabilité des KidZos en fin de la journée. L'activité variait, et Camila a ainsi été tour à tour «à la clinique vétérinaire, chez Gasco, Coca-Cola, Pronto-Matic, au cabinet dentaire, à la mutuelle Los Andes, chez Pizza Hut, chez Pro Arte, ou encore chez Entel et Savory ». Quand il s'agit de décrire son travail, Camila identifie neuf étapes :

1. Convaincre les enfants de venir dans ton activité : être dans la rue et les inviter, leur expliquer combien c'est payé, et ce qu'ils peuvent produire. Chez Gasco, je leur montrais le camion et je leur disais qu'ils allaient monter dedans pour répartir les bidons de gaz. Bref, il fallait trouver des techniques pour attirer les enfants 2. Les enregistrer grâce à leur bracelet et les faire payer si l'activité était «payante ». Il faut toujours les enregistrer pour que les parents sachent où ils sont. 3. Les saluer, et leur expliquer qu'on va leur apprendre à comment être un excellent pompier, médecin ou livreur de gaz: «Kai, bonjour, mon nom est Camila et aujourd'hui je vais vous apprendre à être le meilleur livreur de gaz». 4. Leur faire mettre l'uniforme de travail, afin qu'ils soient prêts pour leur rôle. 5. Leur expliquer l'activité, ce qu'ils vont devoir faire, comment «travailler». Pour certaines marques, on leur montre une vidéo. 6. Leur demander s'ils ont des questions, pour vérifier qu'ils ont bien tout compris. 7. Bien les inclure dans l'activité en s'assurant que tous les enfants participent, les motiver, mais sans jamais les forcer. 8. Conclure l'activité : les remercier, les féliciter pour le travail réalisé et se dire au revoir avec le salut de KidZania: «See you». 9. Les payer si l'activité est rémunérée, et enregistrer de nouveau leur bracelet. Cela permet d'indiquer aux parents [...] l'heure à laquelle ils ont quitté le lieu.

Ces étapes lui ont été présentées lors de la formation et les animateurs doivent les respecter. Leur activité est donc particulièrement codifiée et possiblement répétitive. Ce cadre ritualise aussi les façons de faire relation avec les enfants : les attirer, les faire entrer dans le jeu, les accrocher et les tenir, et enfin les en faire sortir. De fait, il favorise un processus d'enrôlement, que l'on observe dans d'autres contextes d'animation, notamment dans les parcs à thème. Mais, contrairement à Disney où l'enrôlement repose sur «la participation de l'usager à l'univers fictionnel du parc travaill[ant] à réguler ses conduites et à le rendre solidaire d'un certain ordre social » (Clément, 2016, p. 132), à KidZania, il est soutenu par les jeux de rôle qui constituent le 
cadre des activités dans lesquelles les enfants s'engagent. Bref, à la différence des parcs Disney, ce lieu introduit une participation active des enfants à la narration et il ne s'agit plus seulement de rentrer dans une fiction, mais bien de jouer une fiction en endossant un rôle.

Cependant, comme l'explique Camila, ce cadre n'était pas sans poser problème aux animateurs ou tout au moins à certains :

Pour toutes les activités, je cherchais à faire un peu bouger le scénario, pour qu'il soit plus amusant pour les enfants. J'étais toujours soucieuse de ne pas tomber dans la monotonie. Mais à KidZania, normalement, tu ne peux pas faire ça. Mais chaque enfant est unique et il faut pouvoir s'adapter. En plus, si tu ne fais pas ça, tu t'ennuies en répétant comme un perroquet les scénarii. Voilà par exemple ce que je faisais chez Gasco. Avant de commencer, je leur demandais leur prénom. Comme ils n'étaient pas nombreux, c'était facile de les mémoriser : «Kai, bonjour. Mon nom est Camila et je vous souhaite la bienvenue chez Gasco. Aujourd'hui, je vais vous apprendre à être les meilleurs livreurs de gaz. Mais avant, j'aimerais savoir ce que vous savez sur le gaz ? » Là, j'improvisais et posais des questions pour guider un peu les enfants et les inciter à répondre. Ensuite venait le moment de la vidéo : «Je vois que vous en savez beaucoup sur le gaz! Mais comme a dit Matias, le gaz peut être très dangereux si on ne sait pas comment travailler avec. C'est pourquoi je vais vous montrer cette vidéo. Prêtez-y attention, c'est important ». Et je lançais le film. À la fin, je leur posais des questions et j'expliquais quelques mots : «Très bien, qui veut me dire ce qu'il a retenu du film? » Ensuite, on commençait à travailler avec le gaz, en remplissant les bidons et en les fermant. En général, les enfants respectaient bien leur tour et étaient très attentifs. Parfois, certains étaient désordonnées, mais je n'ai jamais eu affaire à des conflits. Quand les bidons étaient prêts, on sortait pour aller les livrer, en utilisant le camion. Les enfants montaient sagement dans le camion, et une fois installés, je leur expliquais les règles de sécurité et le cri du livreur: on changeait souvent, mais il s'agissait toujours de crier «le gaz » sur différents tons et suivant différents rythmes. Certains disaient: " le gaaaaaaaaz », d'autres : « le gaz... le gaz... le gaz... », d'autres encore : « Le gaz!». A chaque arrêt, un enfant descendait avec son bidon, et livrait à une boutique, sans oublier de saluer. Et on continuait de parcourir le parc. Pour cette activité, il y avait deux animateurs : celui qui conduisait et s'occupait des enfants et le second, lui, descendait pour aider l'enfant à livrer. Une fois tous les bidons livrés, on retournait chez Gasco, on se saluait, et je payais les enfants.

Camila déployait donc une série d'astuces, à la limite voire hors du scénario prescrit, pour s'assurer que les enfants s'engagent dans l'activité, pour qu'ils entrent dans le jeu de rôle et pour qu'elle soit en mesure de leur transmettre toute une série d'informations dans un souci éducatif telles qu'elles sont indiquées dans le script: accueil convivial et sécurisant, attention à la singularité de chacun, sollicitations et relances régulières. Sans oublier son souci de la dimension ludique qui n'est pas toujours très présente dans les activités. Soit parce que l'activité est "sérieuse ». Carmen, (cinq ans, parc de Santiago), se souvient ainsi : « Il y a aussi une dame qui nous a parlé de l'eau. C'était ennuyeux, mais pas trop. Et chez les militaires, le prof était assez sérieux, ce n'est pas l'activité que j'ai préférée ». Soit parce que l'activité est particulièrement répétitive. Dans le parc de Dubaï, les trois filles d'une dizaine d'années que j'accompagne sont ainsi très claires sur la police: "C'est ennuyeux: on suit l'animateur qui nous mène dans différents lieux et demande: "Savez-vous ce qu'il faut faire en cas d'urgence?" Et nous on disait "il faut appeler le 999". Par contre les pompiers c'est fun. Et l'hôtel aussi ». Si les enfants s'ennuient par moment je n'ai jamais été témoin d'un abandon: peut-être sont-ils suffisamment «enrôlés »? Peut-être savent-ils que l'activité finira bientôt ? Peut-être enfin les parents, jamais très loin, les 
encouragent-ils à persévérer? Quoi qu'il en soit, les animateurs opèrent un «travail d'intéressement» (Hélou et Lantheaume, 2008) au moyen de stratégies et de ruses qui visent à installer les enfants dans le jeu et à les faire participer. Cela est d'autant plus nécessaire avec certains enfants : «Ceux qui étaient déjà venus plusieurs fois ou qui ont beaucoup de personnalité résistent pas mal à suivre le script. C'est pourquoi je les modifiais, pour que ce soit plus amusant, mais sans jamais le dire à mes chefs ». Dit autrement, pour mener à bien son travail (le travail réel), l'animatrice prend de la distance avec le scénario (le travail prescrit).

Si le pas de côté vis-à-vis du script semble nécessaire dans certaines situations pour que les enfants prennent part aux activités et s'amusent, cet écart est également important pour l'engagement de l'animatrice dans son propre travail. D'abord, cela lui permet de ne pas répéter "comme un perroquet les scénarii » et d'imaginer autre chose, éventuellement avec des collègues. Ensuite, en se déportant de la sorte, Camila est en mesure de transmettre des éléments plus personnels sur lesquels elle s'appuie, et pas seulement les informations indiquées dans les scripts ${ }^{17}$ :

Gasco et la clinique vétérinaire, c'est ce que je préférais. Car je pouvais jouer avec

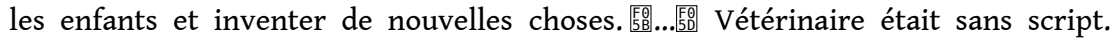
Enfin, il y en avait un, mais je ne le suivais pas. J'ai toujours aimé les animaux, j'ai eu toutes sortes d'espèces chez moi et je pratique l'agility ${ }^{18}$ avec mes chiens, je vais à des salons et des expos. J'adore enseigner aux enfants comment se comporter avec les animaux, et ne pas avoir peur d'eux. [...] Mais c'était avant, quand il y avait encore des animaux vivants à la clinique.

On retrouve ici la définition que la psychodynamique donne du travail : une activité à la fois matérielle et psychique ; et du «travailler » : « un certain mode d'engagement de la personnalité pour faire face à une tâche encadrée par des contraintes matérielles et sociales » (Dejours, 2009, p. 25). De ce point de vue, le travail est avant tout « un rapport subjectif » et il est bien « vivant » $(\text { Ibid. })^{19}$.

Camila, on l'a vu, aime jouer avec les enfants, et cela a compté dans son choix de travailler dans le parc. Mais les observations menées montrent que tous les animateurs ne sont pas autant engagés. Certains semblent parfois s'ennuyer et les échanges informels avec eux, sur le pas de leur local entre deux activités, témoignent de difficultés qu'ils peuvent rencontrer. L'un d'entre eux m'explique ainsi avoir du mal à supporter ceux «qui n'en font qu'à leur tête » et qui, d'après lui, ne sont pas rares. Peut-être ces animateurs ne parviennent-ils pas toujours à prendre de la distance avec le cadre très strict? Peut-être, également, n'arrivent-ils pas à se mettre à hauteur d'enfant et à entrer, comme Camila, dans leur « jeu symbolique » ? Or on le sait :

Une telle participation au jeu de l'enfant [...] nécessite de sortir de la position d'adulte pour entrer dans celle d'un enfant. L'exercice est périlleux en ce sens que l'adulte doit jouer en se conformant au scénario et donc aux attentes de l'enfant (ou des enfants) sans pour autant pouvoir réagir comme un enfant qu'il n'est pas et qu'il ne peut pas être. » (Roucous et Haberbusch, 2010, p. 219)

\section{Éduquer/jouer ? Savoir (re)cadrer le jeu}

Dans les propos de l'animatrice et les observations menées, on voit se dessiner une ambivalence entre activité sérieuse et jeu qui tient au positionnement des parcs, au carrefour de l'éducation et du loisir. Camila le rappelle : «C'est sûr, c'est de l'éducation 臨en監 plus du divertissement. Pour chaque activité, je présentais aux enfants une 
profession en particulier, mais je leur parlais également du travail en équipe, de l'importance du respect de l'autre, et de la nécessité de suivre les ordres d'un supérieur. Mais tout ça en jouant. »

Dans les locaux, les enfants constituent un groupe captif, en présence de l'animateur, et sous le regard souvent des parents qui les observent derrière les baies vitrées. Ils font preuve de concentration et le sérieux domine. Ainsi, dans le studio radio Disney de Santiago, les enfants, après avoir revêtu un gilet au nom de l'entreprise, s'installent autour de la table. Là, le Zupervisors leur présente le rôle d'animateur radio qu'ils vont jouer : après avoir mis leur casque, ils devront répondre tour à tour aux questions qu'il leur posera en lisant le texte inscrit sur leur écran d'ordinateur. Les enfants sont concentrés: ils écoutent attentivement les questions, attendent leur tour pour intervenir au bon moment, puis répondent avec plus ou moins de facilité selon la maitrise qu'ils ont de la lecture. À la fin de l'activité, ils ressortent avec le CD gravé de l'émission. Bien souvent, en introduction des activités, l'animateur donne des indications sur l'entreprise, la marque ou l'institution. Ainsi, dans le local Nestlé, un film est diffusé sur l'histoire de l'entreprise et de Trencito, la barre chocolatée qu'ils vont ensuite fabriquer. Et à la clinique Alemana, l'animatrice avec qui je discute entre deux groupes qu'elle accueille m'explique qu'elle précise aux enfants que cette clinique est l'une des meilleures de la capitale. Les enfants sont donc placés dans une situation où l'adulte leur donne une série d'informations et d'instructions à écouter, à comprendre et à suivre. Pour l'animateur, il ne s'agit pas vraiment de jouer avec les enfants, ni de se mettre à leur hauteur pour entrer dans leur jeu, mais plutôt de leur faire faire des tâches et de leur donner une série d'informations dont le statut est trouble. Certaines concernent les règles du jeu de rôle, d'autres l'entreprise et son produit, d'autres encore sont relatives à un contenu plus éducatif: ainsi, chez le dentiste, les enfants, qui interprètent le rôle d'assistant dentiste, procèdent au diagnostic dentaire d'un faux patient allongé sur un fauteuil (il s'agit d'un mannequin) avant de faire leur autodiagnostic. L'animateur leur explique ensuite l'importance de bien se brosser les dents pour lutter contre les caries.

Malgré cette posture d'exécutant et l'ambiance appliquée, les enfants tirent un certain plaisir des activités. Il tient d'une part à la fabrication ou réalisation de quelque chose de concret: une émission de radio, une pizza ou un décollage; et d'autre part à la satisfaction de se conformer à des rôles issus du monde du travail en sollicitant leur corps en référence au « réel » : courir et ramper à l'entraînement militaire, soigner un patient installé sur un fauteuil de dentiste, faire un reportage en posant une série de questions un calepin à la main, ou encore passer des produits en caisse et faire payer le client. Emilia explique : "J'ai fait une opération et c'était vraiment incroyable, on était sur la personne avec des vrais instruments». Carmen se souvient, elle, de tous les objets qu'elle a manipulés : «Le courrier c'était génial, il y avait un petit caddy qu'on

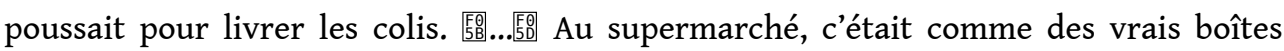
d'aliments, je voudrais trop le refaire ». Ce plaisir relève de ce que Caillois (1967 [1958], p. 80) appelle le ludus, cette façon de jouer qui suppose de suivre la règle ou ici le script qu'on (s')impose et permet de tester certaines habiletés: taper correctement à l'ordinateur, bien laver une voiture ou faire les bons gestes des premiers secours. Dans ce dispositif ludique, la conformation à la règle se réalise à l'occasion de cette forme de jeu qu'est l'imitation (la mimicry dit Callois), qui renforce la fiction et le second degré : les enfants jouent et interprètent le rôle de pompier ou de médecin, tout en sachant 
que le «faux » feu éteint avec de la "vraie » eau ne brûlera pas le parc et que l'acte chirurgical n'entraînera pas la mort du patient.

D'autres fois cependant, c'est le jeu pour le simple plaisir de jouer, plus frivole et désintéressé qui surgit dans le parc: dans les rues quand les enfants s'amusent entre deux jeux de rôle; ou quand, dans le cadre d'une activité, ils procèdent à des sortes d'échappées belles qui les éloignent des adultes et leur permettent de prendre un peu de distance avec le scénario, voire de le détourner, à l'instar de l'activité de livraison de colis évoquée plus haut. Cette forme de jeu relevant du play, et dans sa forme plus radicale de la paida, « embrasse les manifestations spontanées de l'instinct de jeu » et « intervient dans toute exubérance heureuse que traduit une agitation immédiate et désordonnée » (Caillois, 1967 [1958], p. 77). Souvent sanctionnée par les adultes qui y voient du "bazar ", elle se trouve très encadrée dans le parc par les jeux de rôles qui disciplinent les actions des enfants. Quand elle se fait plus insistante, les animateurs y répondent de différentes manières selon l'évaluation qu'ils font de la situation, et selon leur propre capacité à faire avec. Dans certains cas, l'animateur peut lui aussi glisser vers le play, comme celui-ci décrit par Camila :

Chez Coca-Cola [une usine d'embouteillage], plusieurs fois j'ai eu des enfants qui ne faisaient pas toujours ce que je leur demandais. Quand les bouteilles sortaient de la machine, je leur disais d'attendre un peu avant de l'ouvrir. Bien sûr, ils l'ouvraient, et elles explosaient en salissant tout. A la place de m'énerver, je riais avec eux.

Dans d'autres situations, les animateurs cherchent à canaliser cette effervescence dont les frontières avec la «chamaillerie » ne sont pas toujours claires, en mobilisant par exemple des éléments de la culture ludique enfantine. Camila raconte :

Une fois, deux garçons du même groupe se battaient pour être chacun le premier. Il fallait que j'intervienne car ils étaient sur le point de se taper. Je les ai appelés et je les ai fait jouer à « pierre, feuille, ciseaux ». Je leur ai dit que le premier qui gagnait commencerait, et que l'autre devrait attendre son tour.

L'animatrice utilise un jeu bien connu pour recadrer la situation et pour les faire entrer dans l'activité en respectant les règles du parc. Cela n'est pas sans faire penser à ce que Besse-Patin a observé chez les animateurs de loisir éducatifs qui sont animés par une certaine conception du jeu dans ces espaces où, explique-t-il, « les enfants ne sont pas censés jouer selon leurs règles, mais bien apprendre à "jouer correctement” » (2019).

Les animateurs sont donc dans une situation complexe : ils doivent animer une activité à vocation éducative, tout en conservant une part ludique essentielle pour assurer la « jouabilité » de leur activité. Pour tenir ensemble l'activité et le jeu, ils opèrent, de manière fine, une série de cadrages, dé-cadrages, et re-cadrages, qui rendent possibles des engagements différents de ceux qui sont directement proposés par le script. Aussi, ici comme dans d'autres situations de loisirs, « animer un jeu c'est [bien] le remodaliser en lui conférant une signification éducative supplémentaire et l'arbitrer pour "empêcher les joueurs de transformer le jeu en un autre jeu" (Goffman 1991, p. 91) " (Besse-Patin, 2019). L'espace du parc et les objets sont des éléments du dispositif qui participent à ces mises en cadre: l'espace-temps de la file d'attente pour chaque activité, de même que les déguisements, permettent une transition entre deux cadres et engagements : celui de la rue où les enfants jouent de manière plus libre, et celui du local, lieu de l'activité et du jeu de rôle.

L'ambivalence de ce travail de «formalisation animative » (Besse-Patin, 2019) se donne pleinement à voir à l'occasion d'un cas limite, les vols de KidZos. Cette situation m'a d'abord été rapportée par le directeur du parc de Santiago rencontré, non sans 
amusement. Il se souvenait notamment de la fois où une "bande d'enfants " avait chapardé quelques liasses de billets, alors qu'ils devaient, dans le cadre de leur activité, les transporter à la banque. Les enfants, pris la main dans le sac, avaient été réprimandés, puis exclus du parc. Autrement dit, un rappel à l'ordre avait été signifié, visant à sanctionner un acte considéré comme déviant, à savoir le vol de (faux ou pseudo) argent. Pourtant, on pourrait voir dans ce comportement un rappel à jouer de la part des enfants. Dans ce parc où plusieurs dizaines de jeux de rôles sont possibles, les enfants ont imaginé, sans en avoir nécessairement conscience, mais tout en sachant par contre que c'était interdit, un rôle de plus: celui du voleur ou du bandit qui appartient pleinement aux rôles traditionnels de la culture enfantine des bons et des méchants (Brougère, 2003). Ce faisant, ils ont réintroduit une part de la réalité mise de côté par les concepteurs. En outre, comme le souligne Caillois (1967 [1958], p. 38), la triche ne met pas fin au jeu, mais elle peut venir au contraire rappeler que l'on est bien dedans : le tricheur ne ruine en effet pas tant le jeu qu'il remet en cause l'arbitraire voire l'absurde des règles qui le fondent.

Camila, elle aussi, a été confrontée aux vols de Kidzos, forfait souvent réalisé par les plus grands (huit/dix ans et plus). Elle se rappelle un vol en particulier :

Plusieurs fois des enfants ont volé des KidZos. Ils entraient dans le local quand il était vide et ils volaient tous les KidZos [...] Une fois, alors qu'on était dans le camion de Gasco, j'en ai vu deux avec une attitude suspecte. C'était peut-être des voleurs de KidZos. J'ai prévenu ma collègue, qui conduisait le camion. J'ai appelé un autre animateur pour qu'il me remplace, j'ai expliqué la situation aux [autres] enfants, qui ont très bien compris, et je leur ai dit que j'allais me rendre à la Police pour qu'elle attrape ces voleurs. J'ai pris le chemin le plus rapide et j'ai attrapé les voleurs. Je leur ai demandé de me rendre les KidZos et je les ai amenés à la Police, sans qu'ils s'en rendent compte. Là, on les a laissés à la prison quelques minutes, surveillés par des enfants policiers. Ensuite, avec calme, je suis allée les voir pour mettre au clair les choses et faire en sorte qu'ils ne recommencent pas.

Contrairement à la réaction précédente, l'animatrice, dans sa façon de régler cette infraction cherche à rester dans le jeu-fiction. Plus exactement, par le cadrage qu'elle effectue et la performance qui est la sienne de jouer à livrer les enfants à la police, elle qualifie leur acte de jeu, tout en les recadrant/sanctionnant puisqu'ils atterrissent en prison comme s'ils avaient enfreint la loi. Cette attitude est tout en subtilité, et navigue entre le « pour de faux » puisque leur qualité de prisonniers n'a de valeur que dans le cadre de la fiction qui se déroule dans le parc, et le " pour de vrai » puisqu'elle marque la limite sociale que représente le vol dans la réalité. Ce faisant, l'animatrice maintient à la fois le cadre ludique et la posture de l'adulte qui éduque et rappelle qu'on ne triche ni ne vole pas impunément. On retrouve ici des caractéristiques du "jeu symbolique » qui se déploie entre le jeu d'imitation sur le mode du «faire comme si » et le jeu de fiction qui offre une part d'improvisation (Roucous et Haberbusch, 2010). Et c'est bien parce que l'animatrice (s')autorise cette interprétation et crée ainsi un espace de créativité, qu'il peut se déployer, et qu'une forme de liberté est préservée : la sienne, car elle invente une réponse face à la situation, et celle des enfants.

La créativité est cependant fragile dans le parc. Le lieu se présente comme un dispositif très structurant, et les jeux de rôles qui reposent sur des scripts souvent simples et répétitifs favorisent l'enrôlement des enfants, au détriment du travail de fiction et de la prise de décision. Plusieurs parents l'ont d'ailleurs souligné : les enfants suivent parfois automatiquement ce qui leur est indiqué. En outre, selon l'âge (rappelons que le parc s'adresse à des enfants de quatre à quatorze ans environ), l'envie et l'habilité à jouer 
sur un mode fantaisiste et à convoquer l'imagination évolue. Enfin, la créativité dépend des animateurs, de leur désir et de leur talent à jouer à faire semblant alors qu'ils sont des adultes, mais aussi et surtout de l'espace de liberté dont ils disposent dans leur travail. Or, sur ce point, Camila est certaine : les animateurs ont peu de marge et, pour sa part, elle se gardait souvent d'informer ses supérieurs des pas de côté et autres innovations qu'elle introduisait.

\section{Le travail des émotions au cœur de l'animation} savoir la place de la performance dans le travail et le management. Pour Bryman (2004, p. 103-107), le «travail performatif» est en effet l'un des quatre leviers de ce qu'il appelle la "disneyisation »: le salarié doit non seulement interpréter un rôle dans le cadre de son activité, mais son travail est en outre présenté comme un jeu, relevant du « fun $»^{20}$. L'injonction d'amusement apparait de la même manière dans l'entretien mené avec Florencia, animatrice au KidZania de Santiago en charge des anniversaires: «L'idée, c'était que ce soit amusant pour l'animateur, pour ensuite pouvoir montrer que ça l'est aux enfants ». est d'une façon amusante. Cela n'a rien d'évident, surtout quand les cadences sont élevées, comme le note Florencia : «Les week-end, il nous arrivait d'organiser quinze anniversaires dans la journée. C'était un vrai défi de faire en sorte que tout se passe bien, qu'ils s'enchaînent les uns les autres, et tout ça sans s'arrêter de sourire. » Outre la joie et l'enthousiasme, d'autres émotions sont mises en avant, jouées ou surjouées par les animateurs : la gentillesse qui s'exprime à l'occasion des salutations d'accueil ( KKai») et d'au revoir («See you»), ou encore la bienveillance qui les amène à encourager et à féliciter les enfants, par l'introduction de petits mots : « bravo », «c'est très bien ", " applaudissements pour Luis ", etc. S'il importe d'exprimer des sentiments positifs et enjoués, les animateurs doivent, a contrario, maîtriser d'autres émotions, comme l'agacement ou l'exaspération, notamment vis-à-vis des parents qui accompagnent leurs enfants :

C'est un travail où tu es obligé d'être bien émotionnellement. À l'instant où tu entres dans le parc, tu dois oublier tes problèmes pour être capable de jouer ton rôle, de la meilleure manière. Parfois les enfants sont tristes et il faut les rendre heureux, et faire en sorte qu'ils passent un moment agréable. Tu dois gérer des parents énervés sans t'énerver et sans leur manquer de respect. Ce sont les clients, tu ne peux pas t'énerver et répondre, même si tu passes un sale moment. Bref, tu dois faire partie de ce monde heureux où tout ne doit être qu'amusement. (Camila)

Le travail performatif implique ainsi un «travail émotionnel » intense (Hochschild, 1983), au point qu'il s'agit de performer non seulement un rôle, mais des sentiments : «Il fallait faire preuve, devant les enfants et les adultes, de son dynamisme, de sa joie et de sa bienveillance » explique Florencia. La communication de l'entreprise et celle des 
différents parcs sur LinkedIn confirment l'importance du "fun", les photos des animateurs, toujours souriants, étant accompagnées de slogans comme :

- « formation\#développement\#fun ${ }^{21}$ »

- « récompense\#reconnaissance\#fun ${ }^{22}$ ».

44 À la fois ressort du jeu et mode de management, cette performance est au service d'un " commerce du bonheur", caractéristique de ce que Van Maanen a appelé la "smile factory » à propos de Disney (1991, p. 59). «Disneyland n'a-t-il pas été ouvert avec pour but explicite de "créer du bonheur" 23 ainsi que le spécifie son ordre de mission officiel?», rappelle Clément (2016, p.174). Depuis quelques années, cette « happycratie " s'observe plus largement dans le monde du travail (Cabanas et Illouz, 2018), ainsi que dans le secteur de l'éducation, ce qui n'est pas sans effet sur les enfants. Brougère parle ainsi d'une véritable « rhétorique du fun » à l'œuvre : « en s'adressant à l'enfant (directement ou par la médiation d'un adulte), on fait un travail de définition de ce qu'est un enfant. Aujourd'hui, il apparait comme lié au fun et au divertissement, y compris dans les tâches éducatives au sein de la famille et sans doute au-delà, tout au moins dans certaines cultures" (Brougère, 2013, p. 89). L'injonction, désormais mondialisée, d'animateurs heureux de travailler s'accompagne donc de celle d'enfants heureux de jouer et d'apprendre, comme le suggèrent encore les photos promotionnelles des parcs où ces derniers sont souriants, dynamiques et enthousiastes.

Camila conserve néanmoins un souvenir mitigé de ces quelques mois de travail :

J'ai été déçue. Je pensais rencontrer plus de gens comme moi et c'est devenu difficile de continuer à donner la magie que le parc offre et que je venais chercher en tant que cliente. [...] Le côté commercial était important, plus que le jeu. Je me suis aussi rendue compte que beaucoup de superviseurs faisaient le travail par obligation, et que, à quelques exceptions, ça ne leur plaisait pas vraiment. J'ai aussi appris que la magie a ses trucs, et que tout n'était que jeu. Même les machines qu'on utilisait pour les activités n'étaient pas vraiment réelles.

On voit ici la complexité du métier d'animateur en tant que professionnel du jeu qui doit non seulement "donner à jouer" (Roucous, 2006) ${ }^{24}$, mais aussi travailler à jouer alors que, rappelons-le, ce n'est pas toujours «drôle »: le lieu est clos et bruyant, le cadre impose des tâches répétitives, et les interactions avec les enfants et les parents sont parfois compliquées. Sans parler des petits salaires qui caractérisent de nombreux métiers de l'animation, plus encore quand ils sont assurés par des étudiants.

Animer dans ces parcs implique un travail de médiation incessant entre les dispositifs du parc et les enfants et, de ce point de vue, la question des liens à ces derniers semble essentielle. Camila classe d'ailleurs les enfants en trois catégories. Ceux d'abord qui découvrent pour la première fois les activités: ils ont envie d'apprendre et sont attentifs et il est assez facile d'interagir avec eux. Ceux qui connaissent déjà le parc et cherchent à gagner rapidement des KidZos, soit pour acheter des marchandises qu'ils ont déjà repérées, soit dans le cadre d'une compétition avec copains : souvent plus âgés, ils sont peu intéressés par les activités et, d'après Camila, ils sont les moins appréciés des animateurs. Ceux, enfin, avec qui il est possible de parler de choses et d'autres et de faire relation, sans lien nécessairement avec l'activité. Et c'est visiblement ce(ux) qui l'anime(nt) le plus. 


\section{Conclusion} un script tout en aménageant des espaces d'interprétation et de liberté, ils doivent éduquer sans perdre la dimension ludique ; sans compter, bien sûr, la difficulté à se mettre à hauteur d'enfant.

La dimension éducative, on l'a dit, est revendiquée haut et fort par l'entreprise qui reçoit d'ailleurs le soutien des autorités compétentes en la matière dans de nombreux pays où elle est implantée ${ }^{25}$ et établit des partenariats avec des écoles qui visitent le parc avec leurs élèves :

Des psychologues, des professionnels et des experts du jeu ont été consultés pour concevoir chaque activité, afin de s'assurer que les contenus d'apprentissage sont cohérents au niveau national et mondial : la créativité, la réflexion critique, la communication, la confiance, et la collaboration sont pour nous des éléments-clés. Ils nous aident à comprendre et à évoluer dans le monde. À KidZania, l'objectif est d'apprendre à voler de ses propres ailes, à prendre ses propres décisions et à être auto-suffisant : nous jouons, imaginons, faisons des expériences du monde réel. Cela stimule notre pensée créative et renforce l'estime de soi et la confiance en soi ${ }^{26}$.

réalité, comme le souligne Brougère (2013), il bien difficile de démontrer les liens entre jeu et apprentissage. En revanche, une chose est sûre, les « rhétoriques » autour des jeux éducatifs sont aujourd'hui très puissantes, conduisant à « considérer l'enfant comme support d'éducation et ceci à tous les moments de sa vie » (Brougère, 2002, p. 8). C'est aussi ce à quoi prennent part les animateurs de ces parcs, non sans difficulté, mais non sans plaisir dans certains cas aussi.

Dans les parcs de cette entreprise, le contenu éducatif concerne des informations sur le monde du travail, mais aussi des compétences et valeurs spécifiques qui relèvent d'un certain ethos contemporain lié au (néo)libéralisme: la confiance en soi, la communication, la créativité, etc. Cela tient à la présence des entreprises et des institutions qui, selon les concepteurs, sont au service de la représentation d'une ville "réelle ». Mais leur présence est aussi au cœur du modèle économique de l'entreprise : celles-ci paient en effet un loyer au parc et, à Santiago, cette ressource représente environ un tiers des recettes. Du côté des entreprises, leur participation au parc s'inscrit dans une stratégie marketing et publicitaire et parfois, disent-elles, de responsabilité sociale. Le script que suivent les animateurs n'est donc pas seulement un canevas pour le jeu de rôle, mais aussi le support d'une communication d'entreprise et publicitaire.

En outre, le dispositif et les jeux de rôles contribuent à la socialisation des enfants à un modèle de société fondée sur des formes spécifiques de travail et de consommation qui constituent une réalité parmi d'autres : présenter le supermarché comme le lieu où faire ses courses plutôt que de se rendre au marché, ou encore transmettre un certain nombre de gestes comme utiliser de l'argent pour les échanges ou savoir se servir d'un distributeur automatique (Corbillé 2019). Pour Camila, cependant, le contrat de communication est clair : KidZania repose sur la présence de "vraies marques ", et les animateurs tout comme les parents, le savent. Par ailleurs, comme elle le précise, les enfants sont quotidiennement baignés dans le monde de la consommation: par les publicités qu'ils voient sur les écrans, les discussions des adultes autour d'eux, ou encore les sorties dans les centres commerciaux. Reste qu'un tel lieu interroge sur la 
place croissante des acteurs économiques dans les espaces ludiques et éducatifs, quand bien même ceux-ci sont privés.

\section{BIBLIOGRAPHIE}

AUGÉ M. (1997), L'Impossible voyage. Le tourisme et ses images, Paris, Payot \& Rivages, 1997.

AUTHIER J.-Y et LEHMAN-FRISCH S. (2012), « Variations sur un thème : les manières d'habiter des enfants dans les quartiers gentrifiés à Paris, Londres et San Francisco », Métropoles, 11, https:// journals.openedition.org/metropoles/4584.

BATESON G. (1988 [1979]), La Nature et la pensée, Paris, Le Seuil.

BAUDRILLARD J. (1981), Simulacres et simulation, Paris, Éd. Galilée.

BESSE-PATIN B. (2018), « Loisir et éducation. La formalisation éducative des jeux dans un accueil périscolaire ", Revue française de pédagogie, 204, pp. 5-16.

BROUGÈRE G. (1995), Jeu et éducation, Paris, L'Harmattan.

BROUGÈRE G. (2002), « Jeu et loisir comme espaces d'apprentissages informels », Education et sociétés, 10 , pp. 5-20.

BROUGÈRE G. (2003), Jouets et compagnie, Paris, Stock.

BROUGÈRE G. (2005), Jouer/apprendre, Paris, Economica.

BROUGÈRE G. (2013), « Les jouets et la rhétorique de l'éducation », Le sociographe, 1, n 41, pp. 81-90.

BRYMAN A. (2004), The Disneyization of Society, Londres, Sage Publications.

CABANAS A. et ILLOUZ E. (2018), Happycratie. Comment l'industrie du bonheur a pris le contrôle de nos vies, Paris, Premier Parallèle.

CAILLOIS R. (1967 [1958]), Les jeux et les hommes, Paris, Gallimard.

CHOMBART DE LAUWE M.J., BONNIN Ph., MAYEUR M., PERROT M. et DE LA SOUDIÈRE M. (1976), Enfant en-jeu. Les pratiques des enfants durant leur temps libre en fonction des types d'environnement et des idéologies, Paris, Ed. du CNRS.

CHESNEAUX V. (1997), « Une Socio-Anthropologue dans les coulisses de Disneyland Paris », Socioanthropologie, 2, http://journals.openedition.org/socio-anthropologie/36

CLÉMENT T. (2016), Plus vrais que nature. Les parcs Disney ou l'usage de la fiction dans l'espace et le paysage, Paris, Presses Sorbonne Nouvelle.

CORBILLE S. (2018), « Jouer à travailler dans un parc d'attraction pour enfants », Travailler, $\mathrm{n}^{\circ} 39$, 2018, pp. 57-80.

CORBILLE S. (2019), « Troubles dans le jeu : communication marchande et fantasmagorie du capital au cœur d'un parc d'attraction pour enfants ", Questions de communication, $\mathrm{n}^{\circ} 35$, pp. 215-238. 
DEJOURS C. (2009), Travail vivant, Paris, Payot.

ECO U. (1987), La Guerre du faux, Paris, Librairie générale française.

ELSE P. (2009), The Value of Play, London, Continuum International Publishing Group.

FJELLMAN S. (1992), Vinyl Leaves. Walt Disney World and America, Boulder, Westview Press.

GOFFMAN, E (1991), Les cadres de l'expérience, Paris, Minuit.

HÉLOU, C. et LANTHEAUME F. (2008), « Les difficultés au travail des enseignants. Exception ou part constitutive du métier?", Recherche \& formation, 57, pp. 65-78.

HOCHSCHILD A. R. (1983), « Emotion Work, Feeling Rules, and Social Structure », American Journal of Sociology, 85 (3), pp. 551-575.

HORSCHELMANN K. et VAN BLERK L. (2011), Children, Youth and the City, New York, Routledge.

KATZ C. (2004), Growing Up Global : Economic Restructuring and Children's Everyday Lives, Minneapolis, University of Minnesota Press.

LATOUR B. (2007), Changer de société, refaire de la sociologie, Paris, La Découverte.

LUND A. (2014), « Playing, Gaming, Working and Labouring : Framing the Concepts and Relations », TripleC, 12 (2), pp. 735-80.

MARIN L. (1973), « Dégénérescence utopique : Disneyland », in Marin L., Utopiques. Jeux d'espace, Paris, Éd. de Minuit, pp. 297-324.

MCKENDRICK J. H., BRADFORD M. G. et FIELDER A. V. (2000), « Kid Customer ? Commercialization of Playspace and the Commodification of Childhood », Childhood, vol. 7, n 3, pp. 295-314.

MERMET L. et Zaccaï-Reyners N. (dir.), Au prisme du jeu. Concepts, pratiques, perspectives, Paris, Hernann Editeurs, pp. 245- 267.

ROUCOUS N. (2006), « Ludothèque, un territoire de l'enfance », Les Sciences de l'éducation - Pour l'Ère nouvelle, Territoires des enfants, vol. 39, $\mathrm{n}^{\circ} 2$, pp. 15-32.

ROUCOUS N. (2007), « Les loisirs de l'enfant ou le défi de l'éducation informelle », Revue française de pédagogie, 160, pp. 67-73.

ROUCOUS N. et BROUGÈRE G. (1998), « Loisir et éducation. L'apport d'une nouvelle institution : la ludothèque ", Revue française de pédagogie, 124, pp. 91-98.

ROUCOUS N. et HABERBUSCH N. (2010), «Culture enfantine, jouets et jeu symbolique en ludothèque ", in RAYNA S. et BROUGERE G. (dir), Jeux et culture au préscolaire, Paris, INRP, pp. 191-224.

SAVIGNAC E., ANDONOVA Y., LÉNEL P., MONJARET A. et SEURRAT A. (dir.) (2017), Le Travail de la gamification. Enjeux, modalités et rhétoriques de la translation du jeu au travail, Bruxelles, Peter Lang.

SULLU B. (2017), « Geographies of children's play in the context of neoliberal restructuring in Istanbul », Children's Geographies, 16 (2), pp. 169-183.

VAN MAANEN J. (1991), « The Smile Factory : Working at Disneyland », in FROST P. J., MOORE L. F., LOUIS M. R., LUNDBERG C. et MARTIN J. (dir.) (1980), Reframing Organizational Culture, P. Newbury Park, Sage Publications, pp. 58-77.

WRIDT P. (2004), « An Historical Analysis of Young People's Use of Public Playgrounds in New York City. ", Children, Youth and Environments, 14 (1), pp. 86-106. 


\section{NOTES}

1. Mes remerciements à Baptiste Besse-Patin et Nathalie Roucous pour leur précieux conseils bibliographiques et les échanges que nous avons eus.

2. D'après le site internet de KidZania, les quatre parcs installés au Mexique sont gérés par la maison mère. Les 24 autres parcs sont des franchises commerciales c'est-à-dire qu'ils sont exploités par d'autres sociétés liées par un contrat qui les oblige à appliquer un cahier des charges fixant les principes et les formes du parc. Voir https://kidzania.com/en/franchises. Consulté le 8/12/2019.

3. https://kidzania.com/en/the-company. Consulté le 25/06/2019.

4. http://www.kidzania.com/education.php. Consulté le 20/02/2017.

5. http://www.kidzania.com/education.php. Consulté le 20/02/2017.

6. http://santiago.kidzania.com/es-cl/about_kidzania/story. Consulté le 24/09/2017.

7. "Get ready for a better world".

8. Il existe d'autres parcs à thème ayant la forme de mini-villes, notamment Kizmondo et Microville, qui se revendiquent elles aussi du secteur du divertissement éducatif.

9. Selon les parcs, la tranche d'âge varie un peu. À noter que pour chaque activité, une indication sur l'âge minimum requis est indiquée à l'entrée de l'établissement. Certaines activités, qui nécessitent par exemple de savoir lire, ne peuvent être pratiquées qu'à partir de 5/6 ans. En outre, les animateurs tachent de s'adapter aux capacités des enfants.

10. Il est impossible d'accéder à KidZania sans enfant. J'y suis donc allée en famille ou avec des parents qui ont accepté que je les accompagne et que j'observe la façon dont leurs enfants et euxmêmes se comportent.

11. L'enquête de terrain s'intéresse aux activités et jeux de rôles, et non pas aux anniversaires qui peuvent aussi être organisés dans le parc.

12. Notamment le site du groupe (http://www.kidzania.com/en) et le site du parc de Santiago (http://www.santiago. kidzania.com). Je suis également sur LinkedIn plusieurs comptes KidZania dans le monde : India, London, Doha, etc.

13. Le prix des billets pratiqués et la localisation des parcs expliquent largement cela.

14. Il s'agit là des exemples de partenaires privés et publics observés lors des observations de 2016 et 2018. Mais certains ont pu quitter le lieu et être remplacés.

15. Ce passeport, qui permet de devenir KidZanian, fonctionne en réalité comme un programme de fidélité : il existe en effet différentes catégories de passeport plus ou moins chères (à Santiago : "ciudadano naturalizado/distinguido/honorable»), qui permettent ensuite de gagner plus et de dépenser moins à chaque activité. Ce passeport est valable dans tous les parcs de l'entreprise, quel que soit le pays.

16. https://www.youtube.com/watch ? v =iCiDZeu500c. Consulté le 02/10/2017.

17. Les compétences et apprentissages - présumées ou attendues - des enfants sont décrites sur les sites de chaque parc, sur les pages dédiées aux activités. Voir par exemple: https:// santiago.kidzania.com/es-cl/activities.

18. Il s'agit d'un sport canin dans lequel le chien évolue sur un parcours d'obstacles sous la conduite de son maître $»$.

19. Sur ce point voir Corbillé, 2018.

20. La «dysneyisation » est un terme qui exprime la façon dont les principes qui structurent Disney et plus largement les parcs d'attraction se sont étendus au reste de la société, en particulier ce qui concerne la consommation. Les autres leviers de ce processus identifiés par le chercheur sont : la thématisation, la consommation hybride et le merchandising.

21. « \#training\#development\#fuelledbyfun ».

22. «\#reward\#recognition\#fuelledbyfun ».

23. "We create happiness ». 
24. J'emprunte le terme à Nathalie Roucous qui le propose pour rendre compte de la façon dont l'animation est pensée dans le contexte des ludothèques, et qui consiste "à offrir un espace et une présence qui facilite et enrichisse l'activité ludique de l'enfant, [et] se trouve encore renforcée par les possibilités de mettre à leur disposition des objets comme supports de leur activité » (2006, p. 23).

25. A l'ouverture du parc, KidZania Santiago a obtenu de ne pas payer la TVA sur les billets d'entrée en raison de sa mission éducative.

26. Accès: http://santiago.kidzania.com/es-cl/pages/juego-de-rol. Consulté le 05/06/2018, traduction de l'auteur

\section{RÉSUMÉS}

Le secteur dit du divertissement éducatif (edutainement) n'a pas cessé pas de croitre ces dernières années. KidZania, entreprise qui possède une trentaine de parcs à thème dans le monde, propose pour sa part aux enfants de venir «travailler, gagner de l'argent et jouer » dans ses sites qui ont pour forme une mini-ville. Là, grâce à plusieurs dizaines de jeux de rôle, ils y découvriraient le « vrai » monde du travail et acquerraient des compétences. À partir d'une enquête dans les parcs de Santiago du Chili et de Dubaï (observation in situ, entretiens avec des enfants, des parents et des animateurs, et analyses des discours de l'entreprise), il s'agit de comprendre les pratiques des animateurs qui travaillent dans ces parcs, de montrer comment ils procèdent pour faire entrer les enfants dans ces jeux à vocation éducative, et de donner à voir comment ces derniers se saisissent de ces propositions. Le dispositif est aussi étudié pour saisir quel jeu leur est proposé. L'article montre comment les animateurs sont sans cesse amenés à faire un travail de médiation entre les dispositifs du parc, et la réception que peuvent en avoir les enfants, dans une situation traversée par des tensions entre intentions éducatives et dimension de divertissement.

The edutainement sector has been growing in recent years. KidZania, a company with thirty theme parks around the world, offers children the opportunity to come « work, make money and play " in its sites that are shaped like a mini-city. There, through several dozen role-playing games, they would discover the « real » world of work and acquire skills. Based on a qualitiative study in the parks of Santiago de Chile and Dubai (in situ observations, interviews with children, parents and animators, and analysis of the speeches of the company), this paper aims to analyse the practices of the animators who work in these parks, to understand how they proceed to get children into these educational games, and how children deal with these proposals. The device is also studied to see what kind of game is proposed to them. The article shows how animators are constantly led to mediate between the park's devices and the reception that children may have of them, in a situation where there are tensions between educational intentions and the entertainment dimension. 
INDEX

Keywords : theme park, children, role-playing, enrolment, animation, mediation, emotion, education

Mots-clés : parc à thème, enfants, jeux de rôle, enrôlement, animation, médiation, émotion, éducation

\section{AUTEUR}

SOPHIE CORBILLÉ

GRIPIC, Celsa Sorbonne Université 